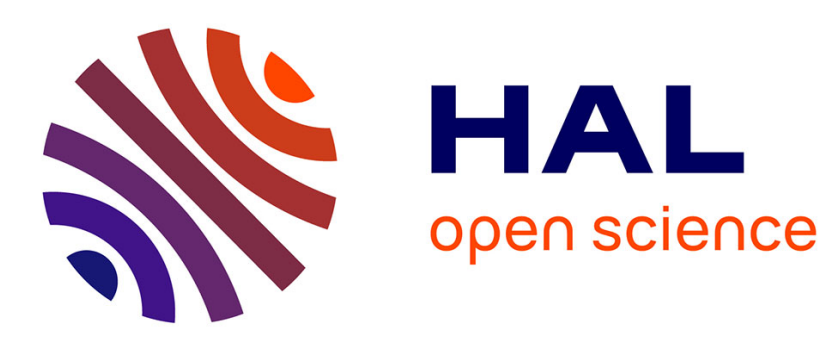

\title{
Observation of long range magnetic order in the reentrant superconductor HoMo6S8
}

\author{
J.W. Lynn, A. Raggazoni, R. Pynn, J. Joffrin
}

\section{To cite this version:}

J.W. Lynn, A. Raggazoni, R. Pynn, J. Joffrin. Observation of long range magnetic order in the reentrant superconductor HoMo6S8. Journal de Physique Lettres, 1981, 42 (2), pp.45-49. 10.1051/jphyslet:0198100420204500 . jpa-00231870

\section{HAL Id: jpa-00231870 https://hal.science/jpa-00231870}

Submitted on 1 Jan 1981

HAL is a multi-disciplinary open access archive for the deposit and dissemination of scientific research documents, whether they are published or not. The documents may come from teaching and research institutions in France or abroad, or from public or private research centers.
L'archive ouverte pluridisciplinaire HAL, est destinée au dépôt et à la diffusion de documents scientifiques de niveau recherche, publiés ou non, émanant des établissements d'enseignement et de recherche français ou étrangers, des laboratoires publics ou privés. 


\title{
Observation of long range magnetic order in the reentrant superconductor $\mathrm{HoMo}_{6} \mathrm{~S}_{8}$
}

\author{
J. W. Lynn (*), A. Raggazoni (**), R. Pynn $\left({ }^{* *}\right)$ and J. Joffrin $\left({ }^{* *}\right)$ \\ (*) Department of Physics and Institute for Physical Science \\ and Technology, University of Maryland, College Park, Md. 20742, U.S.A. \\ $\left.{ }^{(* *}\right)$ Institut Laue-Langevin, B.P. 156 X, 38042 Grenoble Cedex, France
}

(Reçu le 20 octobre 1980, accepté le 1er décembre 1980)

\begin{abstract}
Résumé. - Nous avons effectué des expériences de diffusion de neutrons aux petits angles sur le composé $\mathrm{HoMo}_{6} \mathrm{~S}_{8}$ pour explorer son comportement magnétique au voisinage de la transition réentrante supraconductrice.

Quand on diminue la température de l'échantillon un seul pic de Bragg apparaît en dessous de $T_{\mathrm{M}}=0,75 \mathrm{~K}$ à un vecteur d'onde $Q_{\mathrm{c}}=0,030 \AA^{-1}$; cela prouve l'existence d'une structure magnétique périodique dont la périodicité est de $200 \AA$; quand la température est abaissée une intensité diffusée apparaît aux alentours de $Q=0$ et l'intensité à $Q_{\mathrm{c}}$ décroît : en dessous de $0,71 \mathrm{~K}$ la phase ferromagnétique est obtenue et il n'existe plus de pic dans le domaine $0,002<Q<0,063 \AA^{-1}$.

$\mathrm{Au}$ réchauffement aucun pic n'est observé à $Q$ fini quelle que soit la température. Cependant près de la transition réentrante un léger épaulement apparaît dans l'intensité diffusée pour $\bar{Q} \simeq 0,025 \AA^{-1}$.

Abstract. - High resolution small-angle neutron scattering experiments have been carried out on $\mathrm{HoMo}_{6} \mathrm{~S}_{8}$ to investigate the magnetic behaviour in the vicinity of the reentrant superconducting transition.

With decreasing temperature a single magnetic Bragg peak develops below $T_{M}=0.75 \mathrm{~K}$ at a wavevector $Q_{\mathrm{c}}=0.030 \AA^{-1}$, demonstrating that a transversely-polarized, periodic magnetic structure has formed with a characteristic period of $200 \AA$. With further decrease of temperature additional scattering develops at smaller wavevectors, and the Bragg peak intensity decreases, as the transition to the ferromagnetic state proceeds and superconductivity is destroyed. The spectrum of scattering below $0.71 \mathrm{~K}$ has no peak in the range
\end{abstract}

$$
0.002<Q<0.063 \AA^{-1} \text {. }
$$

On warming from low temperatures no peak is found at finite $Q$ at any temperature. However, near the reentrant superconducting transition there is an enhancement of the scattered intensity for $Q \sim 0.025 \AA^{-1}$.

1. Introduction. - The subject of magnetic superconductors has been revived by the discovery of ternary superconducting materials which contain a periodic lattice of heavy rare earth ions [1]. The conventional spin-depairing mechanism in these compounds is too weak to suppress the superconductivity, and the rare earth ions order magnetically at low temperature. Most of these materials order as compensated antiferromagnets [2], in which case there is no macroscopic magnetic field to disturb the superconducting state. Such systems have provided the first unambiguous examples of the coexistence of superconductivity and long range magnetic order.

On the other hand for ferromagnetic alignment, the macroscopic magnetization associated with the magnetic order will certainly strongly couple these two cooperative states. In terms of the energetics of the system, ferromagnetism prefers parallel alignment (characterized by wavevector $Q=0$ ) of the moments, whereas superconductivity prefers that the magnetization oscillate over a distance which is short compared to a superconducting length such as the penetration depth.

A number of theoretical calculations have been carried out. Generally based on a Ginzburg-Landau equation which couples the superconducting order parameter $\psi$ and the magnetization $\bar{M}$ they result, after minimization of the free energy, in two possible models for the intermediate phase in the temperature region between the superconducting and ferromagnetic phases.

The first model corresponds to $\psi$ uniform and $\bar{M}$ oscillatory; this is the helical state [3-4].

The second model leads to spatial non-uniformity of both $\psi$ and $\bar{M}$ in analogy to the mixed state of a regular type II superconductor : a vortex-like state [5-10] has been proposed.

Both models predict a peak in the magnetic sus- 
ceptibility $\chi(\bar{Q})$ at $\bar{Q}=\bar{Q}_{\mathrm{c}}$ and a magnetization which has a non-zero component perpendicular to $\bar{Q}_{\mathrm{c}}$.

In this paper we describe neutron small-angle scattering measurements $\left({ }^{1}\right)$ on $\mathrm{HoMo}_{6} \mathrm{~S}_{8}$. This material has a superconducting transition $[11,12]$ at $1.8 \mathrm{~K}$, and a magnetic transition $[12,13]$ at $0.67 \mathrm{~K}$, in the vicinity of the reentry to the normal conducting state. At low temperatures the system is a well behaved ferromagnet, but near the reentrant transition a peak in the magnetic scattering in the small wavevector region has recently been observed [12] in a preliminary measurement with a three-axis spectrometer. Qualitatively similar behaviour has been found $[14,15]$ in $\mathrm{ErRh}_{4} \mathrm{~B}_{4}$, which is the only other ferromagnetic superconductor known [16].

2. Experimental procedures. - The neutron measurements were carried out on the D-11 small-angle scattering spectrometer at the Institut Laue-Langevin. This spectrometer utilizes a two-dimensional detector to measure the scattering in a region of reciprocal space around the forward direction $(\bar{Q}=0)$. The range of $Q$ and the resolution may be changed by varying the sample-detector distance and/or the incident wavelength. The ranges of $Q$ used in the present experiment are given in table I. The wavelength $\lambda$ of the incident neutrons is defined by a helical velocity selector, which has a Full-Width Half Maximum (FWHM) wavelength transmission of $9 \%$.

Table I. - Experimental configurations and $Q$ ranges over which usable data could be obtained.

$\begin{array}{cccc}\text { Configuration } & \begin{array}{c}\text { Sample-detector } \\ (\mathrm{m})\end{array} & \begin{array}{c}\lambda \\ (\AA)\end{array} & \begin{array}{c}Q \text { range } \\ \left(\AA^{-1}\right)\end{array} \\ - & - & - & - \\ \mathrm{A} & 2.53 & 14.81 & 0.010 \rightarrow 0.063 \\ \mathrm{~B} & 5.53 & 9.80 & 0.010 \rightarrow 0.045 \\ \mathrm{C} & 10.53 & 5.96 & 0.012 \rightarrow 0.038 \\ \mathrm{D} & 10.53 & 14.81 & 0.005 \rightarrow 0.016 \\ \text { E } & 40.53 & 8.93 & 0.002 \rightarrow 0.008\end{array}$

The powder sample of $\mathrm{HoMo}_{6} \mathrm{~S}_{8}$ was the same as that employed in previous neutron scattering investigations $[12,13,17,18]$. Bulk susceptibility measurements show that this sample has an upper superconducting transition temperature of $1.82 \mathrm{~K}$ and a reentrant transition which occurs at $0.612 \mathrm{~K}$ on cooling and $0.668 \mathrm{~K}$ on warming. The sample was mounted in a cylindrical container whose inner diameter and length were $20 \mathrm{~mm}$ and $3 \mathrm{~mm}$ respectively. The axis of the cylinder coincided with the incident beam direction, and the end faces of the cylinder were sapphire crystals which give very little small-angle scattering. A helium dilution refrigerator was used to cool the sample to temperatures down to $100 \mathrm{mK}$. Thermal contact between the sample and

( ${ }^{1}$ ) This experiment was submitted for beam time allocation to the Scientific Council of ILL in August, 1979 under No 07-01-156. the cold finger was assured by helium condensed into the sample volume. Incident and scattered neutrons traversed the radiation shields through flat, singlecrystal sapphire windows. The sapphire crystals in the radiation shields were coated by evaporation with $6000 \AA$ of aluminum in order to reduce the radiation heating of the sample. The incident beam was restricted in size by a $10 \times 10 \mathrm{~mm}^{2}$ diaphragm placed just before the cryostat to reduce background and improve the resolution.

The scattering at each temperature was always found to be symmetric about the incident beam direction, and therefore the data presented here have been angularly averaged to give intensity as a function of the magnitude of the wavevector $Q$. Generally the statistical uncertainties are negligible for the averaged data.

3. Results. - a) Figure 1 shows the temperature evolution of the scattering on the cooling cycle. These data have been obtained from $Q=0.002 \AA^{-1}$ to $0.063 \AA^{-1}$ by utilizing configurations $\mathrm{A}, \mathrm{B}, \mathrm{C}$ and $\mathrm{E}$ (cf. table I). The background, measured at $2.0 \mathrm{~K}$, has been subtracted. For $0.9 \mathrm{~K} \geqslant T>0.75 \mathrm{~K}$ we found a small increase in the scattering, presumably due to critical fluctuations, which can be described by the customary ferromagnetic correlation function. In particular, there is no peak at finite $Q$. The intensity of this component is too small to see on the figure. At $0.75 \mathrm{~K}$ a sharp peak begins to develop at a wavevector $Q_{\mathrm{c}}=(0.030 \pm 0.001) \AA^{-1}$, with a width which

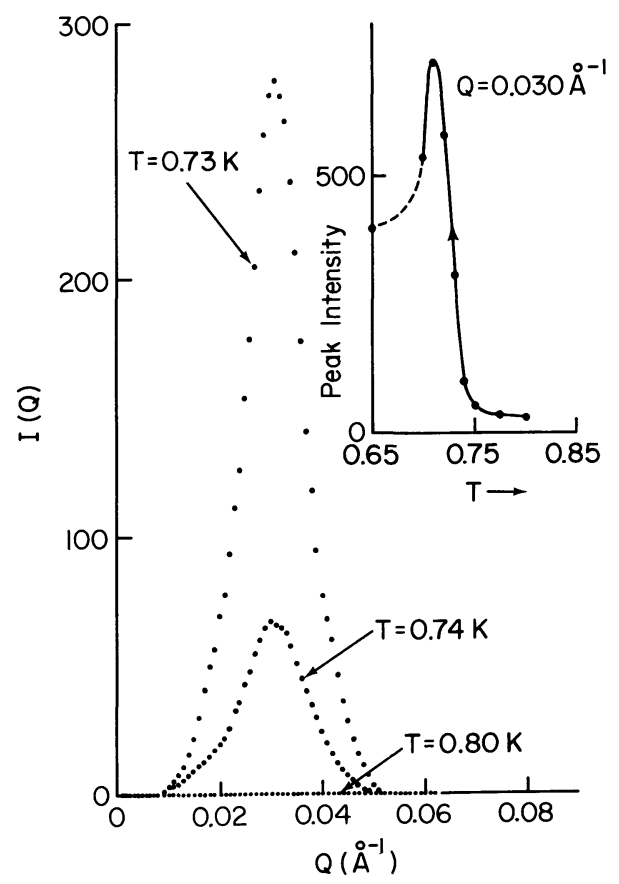

Fig. 1. - Net scattered intensity, after subtraction of the scattering at high temperatures $(2.0 \mathrm{~K})$, showing the rapid development below $0.75 \mathrm{~K}$ of a well-defined peak at a wavevector $Q_{\mathrm{c}}=0.030 \AA^{-1}$. The width of the peak is resolution limited. The inset is a plot of the observed intensity at $Q_{\mathrm{c}}=0.030 \AA^{-1}$. All data were taken during the cooling cycle. 
is limited by the instrumental resolution. The intensity of this peak increases very rapidly at first as shown by the insert in figure 1 . The presence of this peak demonstrates that a long range periodic magnetic state has formed. The period is $2 \pi / Q_{\mathrm{c}} \simeq 200 \AA$, and the distribution of magnetization must be approximately sinusoidal since no higher order peaks are observed. Our best resolution gave an instrumental halfwidth of $0.0047 \AA^{-1}$, from which we deduce that any intrinsic components must be less than $0.002 \AA^{-1}$. Thus the coherence length of the oscillating magnetization exceeds

$$
2 \pi / 0.002 \sim 3100 \AA
$$

or about 15 periods.

With further decrease of temperature, the intensity scattered by the periodic magnetic structure continues to increase as shown in figure 2. Simultaneously, scattering develops at smaller wavevectors, between 0 and $Q_{c}$, and this scattering soon dominates the spectrum. We associate this scattering with the transition to ferromagnetism, and with the apparent concomitant loss of superconductivity. We remark that in comparison with the temperature scale of reference [12], the absolute temperature scale quoted here is higher by $\sim 35$ to $50 \mathrm{mK}$. This is surely due to thermometry, since the identical sample was used in both experiments and there is good agreement between the data sets where they overlap. The absolute temperature scale is only important with regard to relating the measured reentrant superconducting transition temperatures to the neutron scattering results. Clearly it would be desirable to do both measurements together.

b) Figure 3 shows that there is a rather long time constant involved in the system at a temperature of $0.70 \mathrm{~K}$. As the intensity at small wavevectors increases

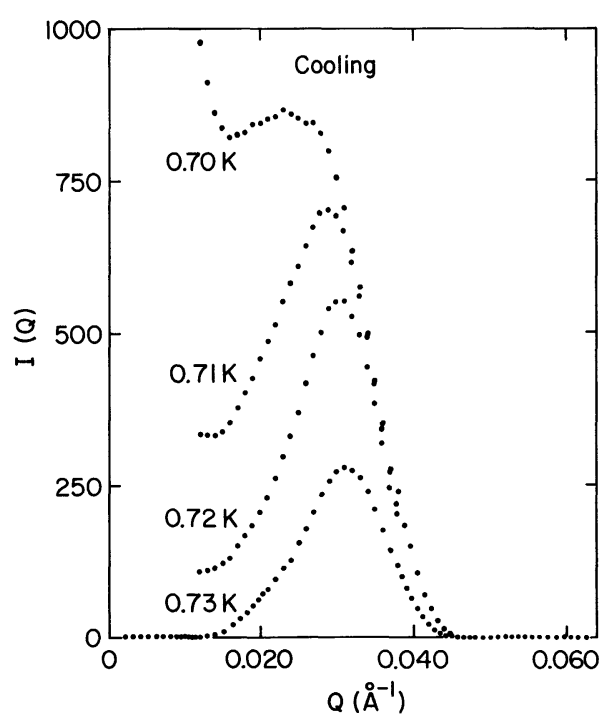

Fig. 2. - Evolution of the scattering during cooling, showing that below $0.73 \mathrm{~K}$ magnetic scattering develops for wavevectors less than $Q_{\mathrm{c}}$.

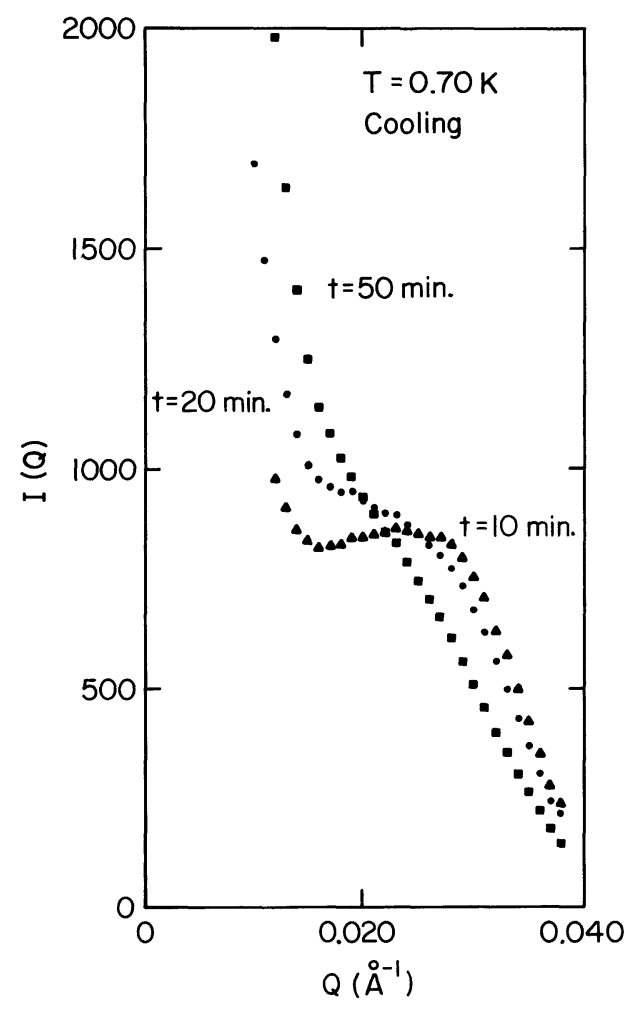

Fig. 3. - The time development of the scattering at $T=0.70 \mathrm{~K}$. The system approaches equilibrium over a period which is considerably longer than at other temperatures. The scattering at smaller wavevectors increases in intensity at the expense of the scattering around $Q_{\mathrm{c}}$.

that associated with the peak at $Q_{\mathrm{c}}$ decreases. This is presumably associated with the transition from the incommensurate oscillatory state to the commensurate ferromagnetic state, accompanied by the reentry to the normal conducting state. We note that the reentrant superconducting transition shows hysteresis. The system takes about an hour to come into equilibrium at this temperature, but the scattering still exhibits a shoulder at $Q \sim 0.025 \AA^{-1}$ when equilibrium is achieved. With further decrease of temperature the intensity of the scattering continues to increase down to the lowest temperature $(100 \mathrm{mK})$, but below $0.69 \mathrm{~K}$ there is no longer any evidence of a peak in the angular distribution of scattering.

c) The results obtained on warming are compared in figure 4 with those obtained on cooling. These data were obtained with a sample-detector separation of $10.53 \mathrm{~m}$ and an incident wavelength of $5.96 \AA$. Figure $4 a$ shows the raw data for a temperature of $0.73 \mathrm{~K}$, along with the data at $2.0 \mathrm{~K}$ which is taken as background. Part $(b)$ of the figure shows the net data after subtraction of the background. We find that there is no peak in the scattering intensity at any finite wavevector on warming. There is, however, an enhancement of the scattering around $Q \sim 0.025 \AA^{-1}$ which is presumably due to a tendency to form an oscillatory magnetization near the superconducting transition. 


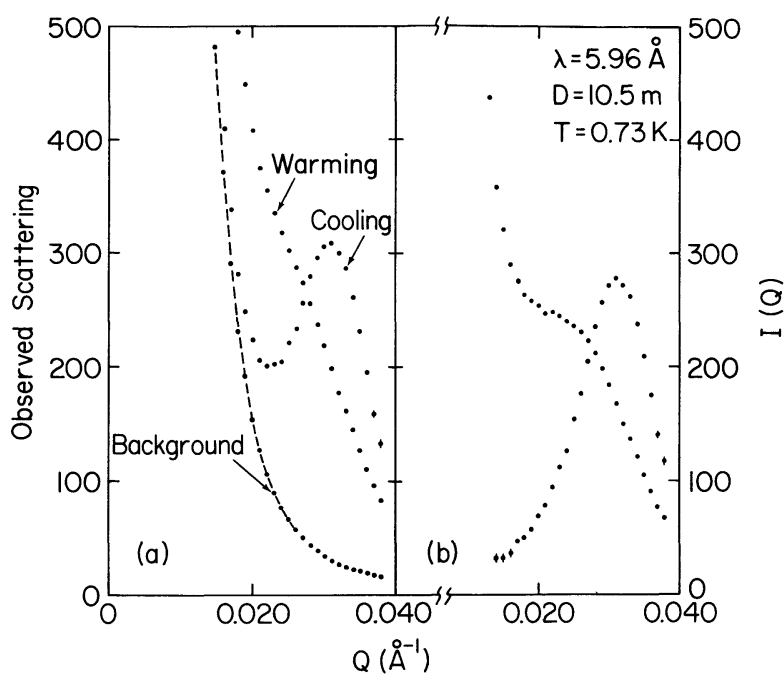

Fig. 4. - Observed scattering spectrum (raw data) at $0.73 \mathrm{~K}$. On cooling the Bragg peak is observed, whereas on warming no such peak is found. The lower portion of the figure shows the net scattering after the high temperature background has been subtracted.

4. Comments. - a) From these experimental results we can conclude without ambiguity that on cooling a periodic magnetic structure develops in the superconducting state. The observed scattering could originate from either a uniform superconducting state and an oscillatory magnetization forming a helical structure ( $\psi$ uniform and $\bar{M}$ oscillatory) or the spontaneous formation of a vortex lattice (both $\bar{M}$ and $\psi$ oscillatory). In the case of a vortex lattice, one would expect, in principle, a series of Bragg peaks [19], rather than the single magnetic peak observed. If there is a second peak at $2 Q_{\mathrm{c}}$ however, its intensity would have to be $<10^{-3}$ of that of the $Q_{\text {c }}$ peak to be consistent with the present experiment. Nevertheless for the high density of vortices that would be required to produce the first peak at

$$
Q_{\mathrm{c}}=0.03 \AA^{-1}
$$

the intensity of the second order $\left(2 Q_{\mathrm{c}}\right)$ peak might well be unobservable [20]. Certainly we can say that the magnetization density producing the observed scattering must be very close to sinusoidal.

b) Another important point is that the observed oscillating magnetization must be transverse to the wavevector $\bar{Q}_{\mathrm{c}}$, since the neutrons couple only to this component of the magnetization. This does not rule out the existence of a longitudinal component $\left(M_{z} \| \bar{Q}_{\mathrm{c}}\right)$; it only establishes that there is indeed a transverse component. We also have no information on the direction of $\bar{Q}_{\mathrm{c}}$ in the crystal since these data were obtained with a powder.

c) For temperatures below $0.7 \mathrm{~K}$ the scattering decreases monotonically with increasing wavevector, and grows in overall strength as the temperature is lowered. Accompanying this intensity increase is of course an increase in the magnetization of the sample in the ferromagnetic state. It is likely that this low temperature scattering originates from domains, which because of the small ratio of exchange to dipole energy are expected to be relatively small in size. Unfortunately little is known at present about the magnetic anisotropy in the system, although in induced moment studies $[17,18]$ the moment appeared to be readily induced in all directions.

Acknowledgments. - It is a pleasure to thank D. Brochier and the low temperature group at ILL for their efforts in preparing and operating the dilution refrigerator. We would also like to thank R. N. Shelton for the loan of the sample, O. Schärpf for performing the aluminum evaporation on the sapphire windows, R. Ghosh for help and advice during the experiment, and R. Currat, D. Saint-James and P. Nozières for helpful discussions. Work at the University of Maryland sponsored by the U.S. NSF, DMR 79-00908.

\section{References}

[1] See, for example, the review by FisCHER, O., Appl. Phys. 16 (1978) 1.

[2] See the reviews by Moncton, D. E., Shirane, G. and ThomLINSON, W., J. Mag. Mag. Mater. 14 (1979) 172;

Moncton, D. E., J. Appl. Phys. 50 (1978) 1880.

[3] Blount, E. I. and Varma, C. M., Phys. Rev. Lett. 42 (1979) 1079.

Bulawsky, L., Rusinov, A., Kulic, M., J. Low Temp. Phys. 39 (1960) 255 .

[4] Ferrell, R. A., Bhattacharjee, J. K. and Bagchi, A., Phys. Rev. Lett. 43 (1979) 154.

[5] Tachiki, M., Matsumoto, H. and Umezawa, H., Phys. Rev. B 20 (1979) 1915 ;

TACHIKI, M., Kotani, A., Matsumoto, H. and Umezawa, H., Solid. State Commun. 31 (1979) 927.
[6] JARIC, M. V. and BeliC, M., Phys. Rev. Lett. 42 (1979) 1015.

[7] JARIC, M. V., Phys. Rev. B 22 (1980) $463 ; 20$ (1979) 4486.

[8] Maekawa, S. and Tachiki, M., Phys. Rev. B 18 (1978) 4688. Makkawa, S., TAChiKi, M. and TaKahashi, S., J. Mag. Mag. Mater. 13 (1979) 324

[9] Kuper, C. G., Revzen, M. and Ron, A., Phys. Rev. Lett. 44 (1980) 1545.

[10] Tachiki, M., Matsumoto, H., Koyama, T. and Umezawa, H., Solid State Commun. 34 (1980) 19.

[11] Ishikawa, M. and Fischer, O., Solid State Commun. 23 (1977) 37.

WoOlF, L. D., Tovar, M., Hamaker, H. C. and Maple, M. B., Phys. Lett. 74A (1979) 363.

[12] Lynn, J. W., Shirane, G., Thomlinson, W. and ShelTON, R. N., to be published. 
[13] Lynn, J. W., Moncton, D. E., Thomlinson, W., Shirane, G. and Shelton, R. N., Solid State Commun. 26 (1978) 493.

[14] Moncton, D. E., McWhan, D. B., Schmidt, P. H., Shirane, G., Thomlinson, W., Maple, M. B., Mackay, H. B. WoOLF, L. D., Fisk, Z. and Johnston, D. C., to be published.

[15] Moncton, D. E., McWhan, D. B., Eckert, J., Shirane, G. and Thomlinson, W., Phys. Rev. Lett. 39 (1977) 1164.

[16] But see Remeika, J. P., Espinosa, G. P., CoOper, A. S., Barz, H., Rowell, J. M., McWhan, D. B., VandenBERG, J. M., Moncton, D. E., FisK, Z., WoOLF, L. D. Hamaker, H. C., Maple, M. B., Shirane, G. and ThomLINSON, W., Solid. State Commun. 34 (1980) 923.
[17] Lynn, J. W., in Crystalline Electric Fields and Structural Effects in f-electron Systems, ed. by Crow, Guertin and Mihalisin (Plenum, N. Y.) 1980 p. 547.

[18] Lynn, J. W. and Shelton, R. N., J. Mag. Mag. Mater. 18 (1980) 1577.

[19] See, for example, Christen, D. K., Kerchner, H. R., SeKuLA, S. T. and Thorel, P., Phys. Rev. B 21 (1980) 102;

Christen, D. K., TAsSet, F., Spooner, S. and MoOK, H. A., Phys. Rev. B 15 (1977) 4506.

[20] See, for example, Saint-James, D., Sarma, G. and Thomas, E., Type II Superconductivity (Pergamon) 1969, p. 72.

Cribier, D., Jacrot, B., Madhav Rao, B., Farnoux, B., Phys. Lett. 9 (1964) 106. 